\title{
FERMILAB ELECTRON COOLING PROJECT: FIELD MEASUREMENTS IN THE COOLING SECTION SOLENOID
}

\author{
C. Crawford, E. McCrory, S. Nagaitsev, A. Shemyakin*, \\ FNAL $^{\bullet}$, P.O.Box 500, Batavia, IL 60510, U.S.A. \\ V.Bocharov, A. Bubley, V. Parkhomchuk, V. Tupikov \\ Budker INP, Lavrentieva 11, Novosibirsk 630090, Russia \\ S. Seletsky, Univ. of Rochester, Rochester, NY 14627, U.S.A.
}

\begin{abstract}
To provide the maximum possible cooling rate for the Recycler Electron Cooling (REC) [1], the cooling section has to be immersed into a high-quality longitudinal magnetic field. Namely, the solenoidal field of 50- $150 \mathrm{G}$ should have an integral of the transverse component below $1 \mathrm{G} \cdot \mathrm{cm}$ over the whole 20-m cooling section [2]. The transverse field components are measured by a dedicated compassbased sensor [3], which has been designed and manufactured at Budker INP, Novosibirsk. The paper describes results of the field measurements performed on the cooling section solenoid prototypes as well as the design and the calibration procedure of the sensor.
\end{abstract}

\section{SOLENOID DESIGN}

The cooling section solenoid proposed for the REC is made up from ten identical modules. Each module consists of the main solenoid, two solenoidal correctors on both ends, 10 pairs of dipole correctors, a cylindrical magnetic shield, and an instrumentation gap between neighbouring solenoids. Some parameters of a solenoid module are shown in Table 1. Two prototypes of the module had been manufactured and measured by a dedicated compass-based sensor [3]. The main goal of the measurements is to align mechanical position of solenoids and adjust currents of correctors to provide an integral of the transverse components below $1 \mathrm{G} \cdot \mathrm{cm}$ over the module length.

Table 1: The basic parameters of the cooling section solenoids.

\begin{tabular}{|l|c|l|}
\hline \hline Solenoid length & 192 & $\mathrm{~cm}$ \\
Solenoid ID & 15 & $\mathrm{~cm}$ \\
Magnetic field & $50-150$ & $\mathrm{G}$ \\
\hline
\end{tabular}

\section{SENSOR}

A scheme of measurements with the sensor is shown in Fig.1. A compass, formed by two cylindrical permanent $\mathrm{NdFeB}$ magnets ( $\varnothing 5 \mathrm{~mm}$ X $10 \mathrm{~mm}$ each), is suspended by a $50 \mu \mathrm{m}$ titanium wire from a cart. A

*Corresponding author. E-mail:shemyakin@fnal.gov

- Work supported by the U.S. Department of Energy under contract No. DE-AC02-76CH03000. laser installed outside the solenoid produces a beam propagating parallel to the solenoid axis. The light is modulated with $50 \mathrm{kHz}$ to eliminate errors caused by a background light. A mirror, attached to the compass, reflects the laser beam to a four-section photodiode. Using the signal from the photodiode, a control system generates currents in two compensation dipole coils wound over the cart. The magnetic field generated by the coils positions the compass such that the reflected light comes to the center of the photodiode. Values of the compensation coil currents in the equilibrium position are used as a measure of external magnetic field transverse components. The cart moves along the cooling section with the precision of $1 \mathrm{~mm}$. Measurements are usually made in points separated by $1-2 \mathrm{~cm}$. After stopping the cart, 10-20 seconds are required to damp mechanical oscillations of the compass and to write data into a file. At the same time, a Hall probe mounted inside the same cart measures the value of the longitudinal component, $B_{z}$.

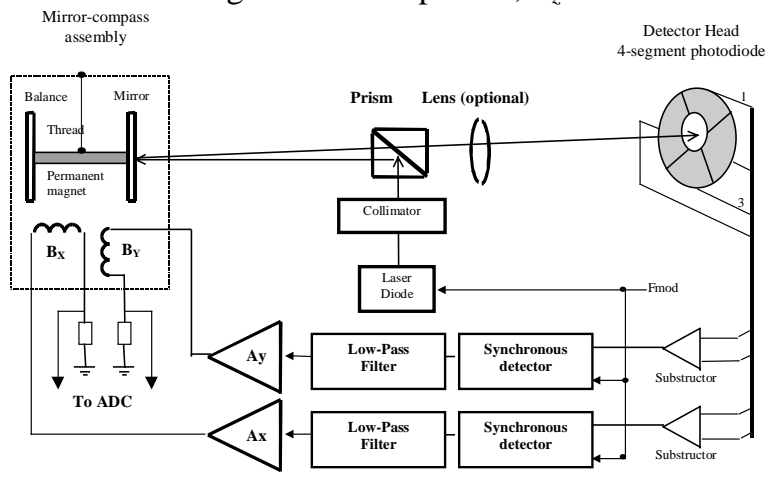

Figure 1: Scheme of the measurement system

\subsection{Sensor calibration}

Since a null method is used, measuring of the compensation coil fields gives the calibration of the sensor. Results obtained by a standard Hall probe were verified by two other methods. First, the solenoid magnetic shields were removed, and a current was passed through a 6-m wire stretched just outside of the solenoid parallel to its axis, the sensor respond was compared with a calculated value. Second, the solenoid was inclined by $1 \mathrm{mrad}$ that resulted in a transverse field change of $10^{-3} \cdot B_{z}$. All three methods were in a reasonable agreement $(\sim 5 \%)$. 


\section{MEASUREMENT ERRORS AND ACCURACY}

The transverse field $B_{i}(i=x$ or $y)$ measured by the sensor was always linear with the longitudinal field $B_{z}(z)$. The value of $B_{i}(z)$ is constituted by several components:

$$
\begin{aligned}
& B_{i}(z)=B_{1 i}+B_{0 i}+\delta B_{i}=B_{z} \cdot\left[\alpha_{i}(z)+\beta_{i}+\gamma_{i}\right]+ \\
& +\left[B_{i}^{\text {earth }}(z)+B_{i}^{\text {shield }}(z)+B_{i}^{\text {sen }}\right]+\delta B_{i}
\end{aligned}
$$

In Eq. (1), $\alpha(z)_{i}$ represents imperfections of the solenoid magnetic field; $\beta_{i}$ is an angle between the solenoid axis and the laser beam; $\gamma_{i}$ is an angle between the magnetic axis of the compass and the normal to the mirror; $B_{i}^{\text {earth }}(z)$ is a field external with respect to the solenoid; $B_{i}^{\text {shield }}(z)$ is a residual magnetic field of the magnetic shield; $B_{i}^{\text {sen }}$ is an offset component originating from misbalance of the sensor, twisting of the compass thread etc.; and $\delta B_{i}$ is randomized fluctuations. Here, $\left\langle\alpha_{i}(z)\right\rangle \equiv 0$ and $<\delta B_{i}>=0$ is chosen zero; $<>$ means an average value in the regular part of a solenoid. Components proportional to $B_{z}$ and constants $\left(B_{1 i}\right.$ and $B_{0 i}$, correspondingly) were separated by measuring of magnetic fields at different solenoid currents. Values of $\gamma_{i}, B_{i}^{\text {sen }}$, and $\delta B_{i}$ represent errors of measurement.

\subsection{Statistical errors and reproducibility}

When the sensor does not move, scattering of measured fields $\delta B_{i}$ is determined by electronics noise and drifts of the laser light caused by air fluctuations. Distribution of errors is close the Gaussian with the value of standard deviation $\sigma_{i}=0.5 \mathrm{mG}$. Measurements made with the cart moving along the solenoid reveal larger scattering $\left(\sigma_{x}=1 \mathrm{mG}, \sigma_{y}=2 \mathrm{mG}\right)$ and a drift of average values (from several $\mathrm{mG}$ during hours to 10 $20 \mathrm{mG}$ after two months). The increase of scattering is believed to be caused by friction in points of thread fastening and by residual mechanical oscillations of the compass. The nature of the drift has not been well understood yet. One of possible reasons can be not the sensor itself but inappropriate mechanical stability of the solenoid supports and/or optical system.

\subsection{Systematic errors}

Non-zero values of $\gamma_{i}$ and $B_{i}^{\text {sen }}$ can result only in an incorrect choice for the average current of dipole correctors. The chosen strategy is minimize the systematic errors as low as possible and, using an information about the electron beam positions in different parts of the cooling section, to adjust the average current of correctors.

The main contribution to $B_{i}^{\text {sen }}$ arises from a misbalance of the compass. This effect was corrected by a balance, an aluminum nut mounted on the compass opposite to the mirror. At $B_{y}^{s e n}>100 \mathrm{mG}$, it changes approximately linear with the nut offset. Reaching $B_{y}^{s e n} \leq 100 \mathrm{mG}$ was possible for both directions only by multiple, almost random attempts. Apparently, the reason is in elastic forces, remaining in the thread (in the present sensor design, the thread should be dismounted for nut adjusting). Typically, $B_{i}^{\text {sen }}$ components are larger than the external fields.

The angle between the magnetic axis of the compass and the normal to the mirror, $\gamma_{i}$, can be very high due to an angle between magnetic and mechanical axes of the permanent magnet cylinders. The latter is found to be about $10 \mathrm{mrad}$. To decrease $\gamma_{i}$, the mechanical angle between the mirror and the permanent magnet enclosure was made adjustable. Such adjustments were made until the measured sum $\left(\gamma_{i}+\beta_{i}\right)$ was close to a precision of the solenoid mechanical alignment. Then, the sensor was rotated by $180^{\circ}$ over $\mathrm{z}$-axis, and the measurements were repeated. If the mirror is not disturbed during the rotation, $\gamma_{i}$ changes its sign only while the solenoid inclination angles $\beta_{i}$ remain the same and, therefore, the value of $\gamma_{i}$ can be calculated. The second rotation by $180^{\circ}$ returns the sensor into its initial position. Comparison of $\gamma_{i}$, measured with this rotation, to the previous value gave an estimation of reproducibility of about $\pm 0.2 \mathrm{mrad}$.

\section{FIELD MEASUREMENTS}

\subsection{Magnetic shielding}

To avoid the influence of an external magnetic field, the solenoid is shielded by two layers of a highpermeability alloy. Each layer is formed by two semicylinders of $1 \mathrm{~mm}$ thickness. The shielding efficiency $K_{\text {shield }}$ was measured with a long wire, also used for the sensor calibration. The value of $K_{\text {shield }}$, determined as a ratio of the magnetic field changes measured without and with the shield for the same wire current, was found equal to 1000 in the plane of the semi-cylinders' joint and to 500 in the perpendicular direction. Hence, the influence of the Earth field in the regular part of the solenoid is negligible $(\leq 1 \mathrm{mG})$.

\subsection{Solenoid transverse field}

The measured transverse field of a solenoid prototype is shown in Fig.2. Non-straightness of the field is $\pm 1 \mathrm{mrad}$. The second pair of curves in Fig. 2 represent measurements performed after rotation of the 
solenoid module by $180^{\circ}$ over z-axis (the component $B_{l y}$ is shown after subtraction of its average value and changing its sign).
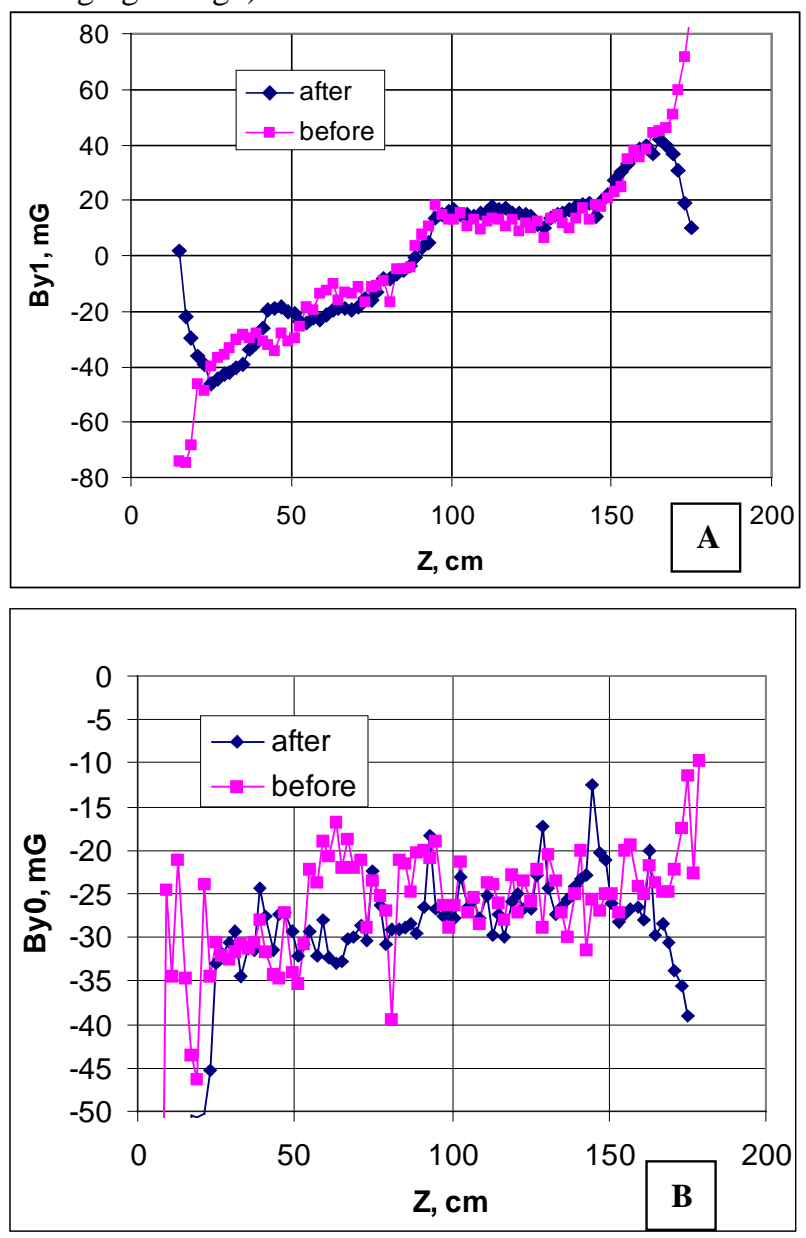

Figure 2: The Y component of a solenoid transverse field, measured before and after a rotation of the module. A and B represent components proportional to $B_{z}$ and a constant, correspondingly. $B_{z}=50 \mathrm{G}$, all correctors are off.

The closeness of curves in Fig.2A proves that the solenoid body is rigid and the sag causing the curve slope originated during the manufacturing. Values of $B_{0 y} \quad$ (Fig.2B) are close as well while the magnetic shield was rotated together with the solenoid. Hence, the shield remnant fields are below $10 \mathrm{mG}$, and the measured $B_{0 y}$ value is determined by a sensor imbalance.

Fields in the same solenoid after setting corrector currents to optimum are shown in Fig. 3. Integrals of the fields over the regular part of the solenoid are less than $0.3 \mathrm{G} \cdot \mathrm{cm}$.

\subsection{Gap between solenoids}

The strongest effect of an instrumentation gap between solenoids is a variation of a longitudinal field. It is compensated by solenoidal coils placed at each end of the module. The currents in the correctors were chosen according to the Hall probe measurements to provide equality of an integral of $B_{z}$ over the gap to the integral over the same length in the regular part of the solenoid.

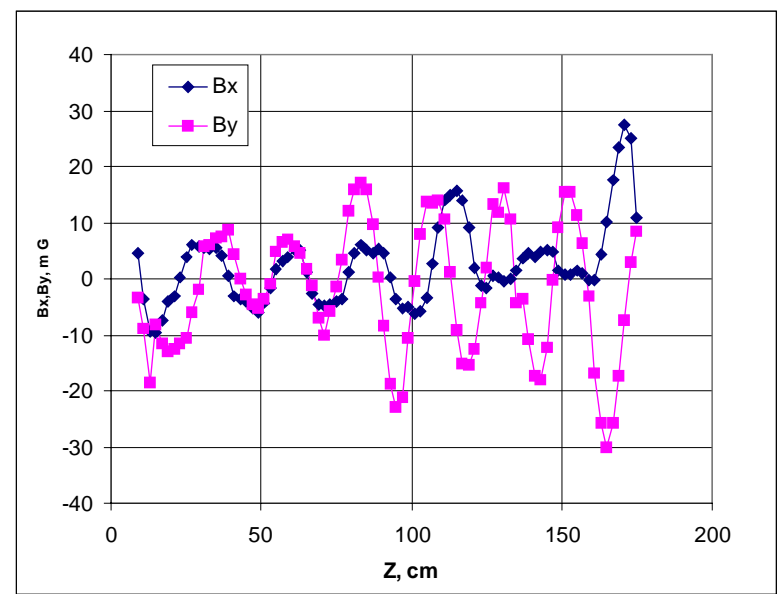

Figure 3: Transverse field with all corrector currents at optimum settings. $B_{z}=50 \mathrm{G}$.

The next step is to compensate the dipole field integral in the gap. The field is composed by a radial fields caused by the $B_{z}$ variation, by a misalignment of solenoid axes, and by an inclination and a shift of the end corrector coils. Contributions of various components were measured by the changing currents separately in each element. The end coil inclination was found 3-7 mrad, and a transverse field generating at optimum longitudinal correction was large. This drawback will be eliminated.

\section{CONCLUSION}

1. The compass-based sensor can measure the solenoid transverse field with a relative accuracy of several mG. Absolute precision, determined by an angle between the magnetic axis of the compass and the mirror, is about $20 \mathrm{mG}$ in a 100 $\mathrm{G}$ longitudinal field.

2. Quality of measured solenoid prototypes is satisfactory for the REC purpose. Integrals of transverse fields can be made below $1 \mathrm{G} \cdot \mathrm{cm}$ at the solenoid field of $150 \mathrm{G}$, if corrector currents are in optimum.

Authors are thankful to A. Makarov for solenoid designing and to all REC group for the help with preparation of the measurements.

\section{REFERENCES}

[1] S.Nagaitsev et al., NIM.-A441(2000) 241-245

[2] S.Nagaitsev et al., Cooling Section Solenoid for the $5 \mathrm{MeV}$ Fermilab Electron Cooling Proc. of EPAC'00, Vienna, 26-30 June 2000, pp. 2447-2449.

[3] V.N.Bocharov et al., Sensor for a precise measurement of the magnetic field direction, XVII ${ }^{\text {th }}$ Workshop on Particle Accelerators, Protvino, Russia, Oct. 17- 20 (in Russian). 